\title{
Sensitizing Solid-State NMR Spectroscopy for the Characterization of Pure and Formulated Pharmaceuticals
}

\author{
Anuradha V. Wijesekara, ${ }^{a}$ David A. Hirsh, ${ }^{a}$ Scott L. Carnahan, ${ }^{a}$ Amrit \\ Venkatesh, ${ }^{a}$ Joseph W. Lubach, ${ }^{b}$ Karthik Nagapudi, ${ }^{b}$ Aaron J. Rossini ${ }^{a}$ \\ alowa State University, Department of Chemistry, Ames, IA, USA. \\ ${ }^{b}$ Genentech Inc., South San Francisco, CA, USA. \\ Email Contact: arossini@iastate.edu \\ Abstract: Solid-state NMR spectroscopy is a workhorse technique for the
} characterization of active pharmaceutical ingredients (APIs) in both pure and dosage forms. Chemical shifts, dipolar coupling constants and quadrupolar coupling constants are all sensitive probes of molecular structure and crystal symmetry. However, solid-state NMR spectroscopy is hindered by poor sensitivity because of the intrinsically small nuclear spin polarization and long longitudinal relaxation times often encountered in the solid-state. Solid-state NMR experiments on dosage forms of APIs are especially challenging because NMR signals from the API often overlap with those from the excipients and the API loading is typically low (1-10 wt.\%).

This contribution will describe how state-of-the-art NMR technologies of dynamic nuclear polarization (DNP) or fast magic angle spinning (MAS) can provide order of magnitude improvements in the sensitivity of solid-state NMR experiments on pure and formulated APIs. ${ }^{1}$ These technologies enable advanced solid-state NMR experiments that improve spectral resolution and provide access to unique structural constraints. For example, with DNP or fast MAS experiments it is possible to measure heteronuclear dipolar couplings between hydrogen and oxygen or nitrogen. Dipolar couplings provide accurate measurements of internuclear distances, enabling hydrogen atoms to be accurately located. ${ }^{2,3}$ Both DNP and fast MAS techniques can also be applied to rapidly detect, characterize and quantify APIs within formulated drug products with low API loadings. DNP enables the rapid acquisition of ${ }^{13} \mathrm{C}$ and ${ }^{15} \mathrm{~N}$ solid-state NMR spectra of dilute APIs in formulations. Finally, it will be shown that fast MAS ${ }^{1} \mathrm{H}$ solid-state NMR spectroscopy provides sensitivity exceeding that of ${ }^{13} \mathrm{C}$ CPMAS solid-state NMR and that simple ${ }^{1} \mathrm{H}$ NMR experiments can be used to rapidly detect dilute API phases within dosage forms.

\section{References}

(1) Zhao, L.; Rossini, A.; et al., J. DNP-Enhanced Solid-State NMR Spectroscopy of Active Pharmaceutical Ingredients. Magn. Reson. Chem. 2018, 56, 583-609.

(2) Zhao, L.; Rossini, A. J.; et al., Characterization of Pharmaceutical Cocrystals and Salts by Dynamic Nuclear Polarization-Enhanced Solid-State NMR Spectroscopy. Crystal Growth \& Design 2018, 18, 2588-2601.

(3) Carnahan, S. L.; Rossini, A. J.; et. al., Probing O-H Bonding through Proton Detected 1H170 Double Resonance Solid-State NMR Spectroscopy. J. Am. Chem. Soc. 2019, 141, 441450.

(4) Hirsh D.A.; Wijesekara, A.V.; Rossini A.J.; et al., Rapid Characterization of Formulated Pharmaceuticals Using Fast MAS ${ }^{1} \mathrm{H}$ Solid-State NMR Spectroscopy, 2019, Submitted for Publication. 